\title{
On the Application of Zone Flooding in a Traffic Warning System
}

Lars M. Kristensen and Kenneth-Daniel Nielsen

DAIMI PB - 573

November 2004
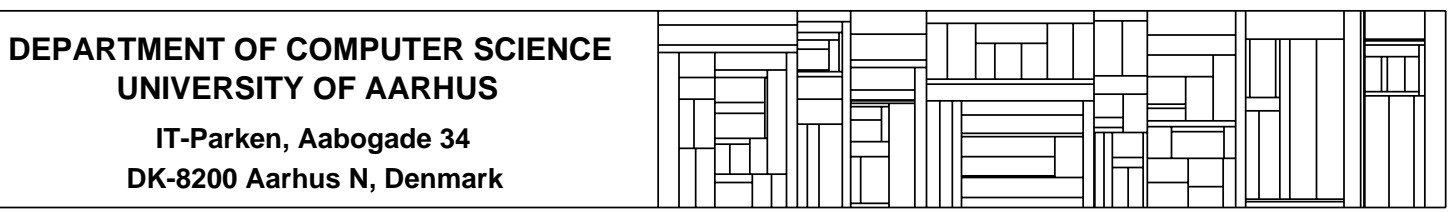


\title{
On the Applization of Zone Flooding in a Traffic Warning System
}

\author{
Lars M. Kristensen* and Kenneth-Daniel Nielsen \\ Department of Computer Science, \\ University of Aarhus, DENMARK \\ Email: \{lmkristensen,kenneth\}@daimi.au.dk
}

\begin{abstract}
The LIWAS Traffic Warning System aims at providing early warning to vehicles about slippery conditions on the road. The LIWAS system is currently under development and consists of two main parts: sensors for measuring and classifying the state of the road, and a communication infrastructure for distributing road-state information to vehicles. This paper concentrates on the communication infrastructure, and considers the application of zone flooding for implementing the distribution of road-state information. Zone flooding combines flooding and geocasting to distribute road-state information in a geographically bounded area. To evaluate the applicability of zone flooding in the LIWAS system, a simulation model has been created using the Network Simulator 2. The simulation model captures a representative road-scenario and has been used to evaluate several flooding protocols when used to implement zone flooding. The primary evaluation criteria are the load on the network and the capability to warn other vehicles in time.
\end{abstract}

\section{Introduction}

Traffic warning and information systems is a promising application area for ad-hoc networking [12] and several research projects are concerned with the development of inter- and intra-vehicle communication infrastructures to enable such systems. Examples of this include the Virtual City Protocol [1], CarNet [2], FleetNet [5], INVENT [7], and TrafficView [4]. These projects cover a broad spectrum of applications and range from development of application software to network protocols and data-link technologies.

The LIfe WArning System (LIWAS) [10] is a traffic warning system based on sensors that are capable of determining whether the surface of the road is dry or is covered with water, snow, or ice. The development of the LIWAS system started in 2003 and has until now primarily focused on the development of the underlying sensor technology. A LIWAS sensor is based on measuring the reflection from light sources when projected on the surface of the road. A vehicle equipped with a LIWAS sensor is able to inform the driver about the state of the road being passed. This can help the driver take precautions according to the current road conditions. Besides this intra-vehicle communication, wireless communication between vehicles will be used to support vehicles equipped with the LIWAS system to inform each other about the state of the road being approached. To support this inter-vehicle communication of road-state information an infrastructure is required.

\footnotetext{
${ }^{*}$ Supported by the Danish Natural Science Research Council.
} 
The LIWAS communication infrastructure can be realised in many ways ranging from a centralised architecture based on, e.g., GSM or GPRS networks combined with Web servers, to a decentralised architecture based on ad-hoc networking and multi-hop communication between vehicles. The two architectures have their pros and cons. The centralised architecture has an advantage in coverage, but a potential problem with scalability. The decentralised architecture has an advantage in scalability, but may have a potential problem when the density of vehicles equipped with the LIWAS system is low. In the LIWAS project it was decided to first investigate how the decentralised architecture based on inter-vehicle ad-hoc communication could be implemented.

Vehicles equipped with a LIWAS sensor continuously make observations about the state of the road they are passing. These observations must be distributed to other vehicles, but only in a limited geographical area around the origin of the observation. Distribution of observation in a limited geographical area is required to reduce the load on the communication infrastructure and to avoid distributing the observations hundreds of miles away to vehicles that will never enter that section of the road or will not enter it until much later. The geographical region where the observation is distributed should not be too large since that would put unnecessary load on the communication infrastructure. On the other hand, it should not be too small as the vehicles need to be warned in time before entering, e.g., a slippery section of the road. The above considerations led to the concept of zone flooding that uses a flooding protocol to disseminate observations about the road-state, and uses geocasting [6] to limit the dissemination to a geographical zone around the location of the observation. Zone flooding can be viewed as a special case of flooding-based geocasting [8] where the source is located in the geocast zone.

Zone flooding can be implemented with many different flooding protocols. This paper presents our result on evaluating several candidate flooding protocols in the context of zone flooding and the LIWAS system. For this purpose we have developed a simulation model using the Network Simulator 2 (NS-2) [11]. Flooding-based geocasting and flooding protocols have also been evaluated in $[3,8,14]$. The mobility scenarios considered in the papers $[3,8,14]$ are based on a square area where nodes moves and communicate at random. These mobility scenarios are not representative for typical roads, and it is therefore of interest to evaluate zone flooding in more representative mobility and communication scenarios prior to implementation and deployment of the LIWAS system. The simulation model that we have developed represents a typical road-scenario where the LIWAS system could be deployed. Simulation is the only manageable approach for large-scale evaluation of the LIWAS system prior to actual deployment as the orchestration of vehicles in a realistic scenario will be extremely difficult.

This paper is organised as follows. Section 2 gives an introduction to the LIWAS system and the communication infrastructure, and it gives an overview of the NS-2 simulation model. Section 3 presents the basic ideas in each of the flooding protocols being evaluated. Section 4 presents the simulation results obtained. Finally, section 5 sums up the conclusions and gives directions for future work. The reader is assumed to be familiar with the basic ideas of ad-hoc networking.

\section{LIWAS Simulation Model}

Figure 1 shows the basic operation of the LIWAS system in a typical road scenario. The figure shows a road with two opposing lanes and an icy section (indicated in gray) crossing both lanes. Vehicles on the road are represented as black dots. Vehicles in the top lane travels towards the left, whereas vehicles in the bottom lane travels to the right. Vehicles passing the icy section will detect ice and pass this observation on to other vehicles. As an example, when vehicle A detects that there is ice on 


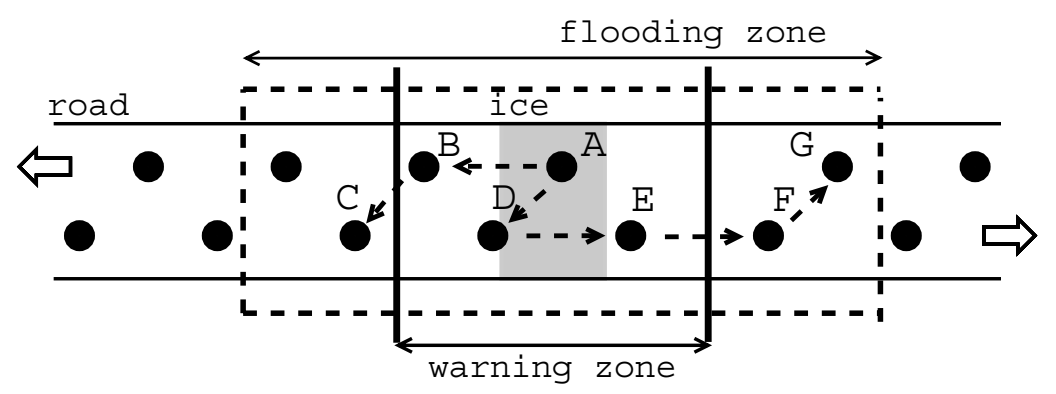

Figure 1: The LIWAS System on a typical road scenario.

the road, this observation will be passed to vehicle $B$ that in turn passes the observation to vehicle $C$ (and so on). In this way, vehicles will be aware of the icy section when approaching it, and hence be able to take the necessary precautions in advance. The vehicles $D, E$, and $F$ travelling in the opposite direction of $A$ can be used as intermediate hops for warning vehicle $G$ approaching the icy section from the right.

Observations about the icy section are of interest in the bounded geographical area determined by the flooding zone indicated by the dashed box in figure 1. The flooding zone contains the vehicles that are expected to pass the icy section soon and which should therefore receive the observations made by vehicles passing the icy section. The receivers of a given observation are therefore defined by their graphical location. In section 3 we present several flooding protocols that can be used to implement the distribution of observations to vehicles located in the flooding zone. We assume that vehicles are equipped with a Global Positioning System (GPS) device that enables them to record the location of an observation, and determine whether they are inside or outside the flooding zone of a given observation.

It is not sufficient that a vehicle is informed about the icy section just before passing it, as there must be sufficient time for the driver to react and take the necessary precautions. For this reason we introduce the warning zone. The boundaries of the warning zone is shown as solid vertical lines in figure 1 and determines the required minimal distance to the icy section when the vehicle receives an observation about the section being icy. This distance has to be large enough for the driver to react in time. Clearly, the warning zone is always contained in the flooding zone for an observation.

The developed NS-2 simulation model mimics the traffic scenario shown in figure 1. Figure 2 depicts the scenario as captured in the simulation model and shows the main parameters of the model. Two lanes comprise the entire network area with a length of 2000 meters and a width of 10 meters. Nodes (vehicles) move along the two lanes. In the basic simulation setup, nodes are distributed evenly on the two lanes, i.e., with the same distance between vehicles. This distance is referred to as the node interval. In section 4 we investigate also scenarios with a random distance between nodes. The duration of the simulations corresponds to 150 seconds of real time. An icy section with a length of $20 \mathrm{~m}$ spans the entire road from one roadside to the other.

The application layer of the simulation model represents a LIWAS sensor that measures the state of the road every 0.5 seconds. This means that nodes going more than $40 \mathrm{~m} / \mathrm{s}(144 \mathrm{~km} / \mathrm{h})$ can pass the icy section of $20 \mathrm{~m}$ without noticing it. We therefore keep node speed below $40 \mathrm{~m} / \mathrm{s}$. When a node measures ice on the road it immediately broadcasts a packet to all its neighbours. The User Datagram Protocol (UDP) is used as transport layer, and each UDP packet contains an observation, i.e., state of the road as measured, and the location of the observation. The zone flooding protocols presented 


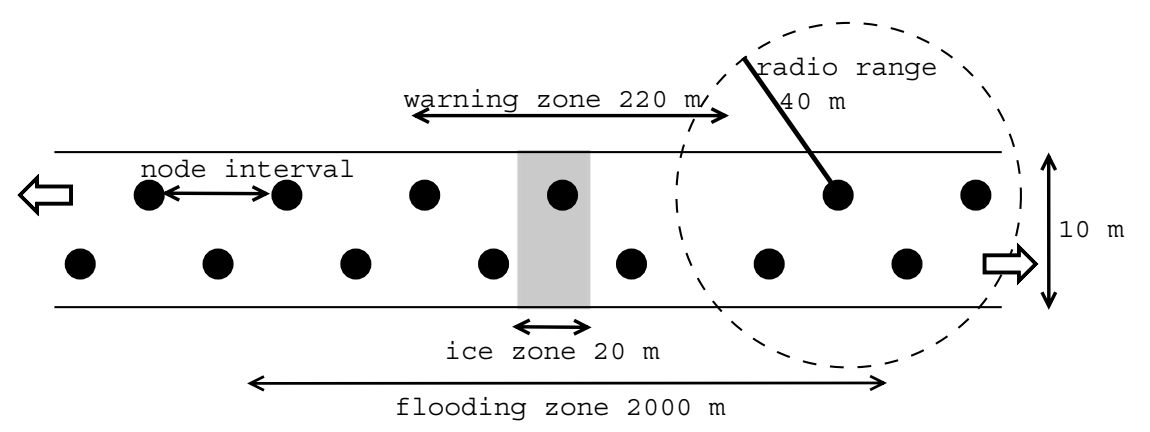

Figure 2: NS-2 simulation model and scenario parameters.

\begin{tabular}{ll|ll}
\hline \multicolumn{2}{c|}{ Fixed parameters } & \multicolumn{2}{c}{ Variable parameters } \\
\hline \hline Network area & $2000 \times 10$ meters & Number of nodes & {$[88.400]$} \\
Icy section & $20 \times 10$ meters & Node interval & {$[0 . .50]$ meters } \\
Warning zone & $220 \times 10$ meters & Speed & {$[10 . .30] \mathrm{m} / \mathrm{s}([36 . .108] \mathrm{km} / \mathrm{h})$} \\
Measuring rate & 0.5 seconds & Flooding zone & {$[200 . .1000]$ meters } \\
\hline MAC protocol & IEEE 802.11 & & \\
Radio range & 40 meters & & \\
Packet size & 64 bytes & & \\
Bandwidth & 1 Mbit/s & & \\
Propagation model & free space & \\
\hline \hline
\end{tabular}

Table 1: Summary of scenario parameters for the simulation model.

in section 3 are implemented on top of the IEEE 802.11 MAC protocol. The 802.11 protocol is set to broadcast mode, which means that channel reservation and acknowledgement are suppressed. The transmission power was set such that the radio range is 40 meters. Packet size was set to 64 bytes and the bandwidth to $1 \mathrm{Mbit} / \mathrm{s}$. We have used the free space propagation model in NS-2. The model has been developed such that different flooding protocols can be evaluated.

Table 1 summarises the parameters of the simulation model. The warning zone is defined such that it extends 100 meters to each side of the icy section requiring vehicles to be warned 100 meters prior to entering the icy section. The number of nodes varies depending on the node interval, i.e., the density of the vehicles. The parameters for the flooding protocols are discussed in section 3 .

The performance metrics used for the evaluation of zone flooding are the number of transmissions and the percentage of warned nodes. Transmissions refers to the number of broadcasts in the network. This equals the transmissions from the nodes that initiates flooding and transmissions corresponding to rebroadcast of packets (observations). Warned nodes is the percentage of nodes that passes the icy section in the course of the simulation and were informed about the icy section before entering the warning zone.

\section{Zone Flooding Protocols}

Zone flooding can be implemented using various flooding protocols, and several flooding protocols have been proposed in the literature (see e.g., $[3,14]$ for a survey). These flooding protocols use different techniques to reduce the number of redundant broadcasts and thereby alleviate the broadcast 
storm problem [3]. In this section we briefly present the flooding protocols selected as candidates for implementing zone flooding in the LIWAS system. The reader is referred to $[3,14]$ for a more complete descriptions of the protocols. All the flooding protocols presented use sequence numbers to ensure termination of the flooding procedure. When receiving a packet the nodes use their assumed GPS device to check whether they are outside the flooding zone of the received observation. If this is the case the packet is discarded, otherwise it is processed according to the flooding protocol being executed.

Simple flooding implements flooding in its most basic form. A source node initiating flooding starts by broadcasting the packet to all its neighbours, and a node receiving the packet, rebroadcasts the packet exactly once. Simple flooding has been included in the suite of protocols mainly as a reference protocol.

Probabilistic flooding uses a probability for rebroadcast of received packets. If the node density is high, a low probability will give less redundant rebroadcasts without harming delivery effectiveness. On the other hand, if the node density is low, the probability must be higher.

Counter-based flooding introduces a Random Assessment Delay (RAD) indicating the time a node has to wait before it rebroadcasts a packet. The RAD is chosen randomly from a uniform distribution between 0 and Tmax. The first time a node receives the packet, a RAD timer is started. The node counts every time the same packet is received by looking at the originator address and the sequence number. The packet will not be rebroadcast if the packet count reaches a predefined counter threshold before the RAD timer expires. If the RAD expires and the count for the packet is below the counter threshold, the packet is rebroadcast.

Distance-based flooding also uses a RAD timer, but with a distance threshold instead of a counter threshold. When a node receives a previously unseen packet it computes the distance to the node that broadcasted the packet. If the distance is larger than the distance threshold, a RAD timer is started and the packet is cached. As long as the RAD timer runs, the distance to transmitting neighbours that broadcast the same packet is compared with the distance threshold. If one of the distances is less than the threshold the packet is discarded. If the RAD timer expires and the packet was not discarded it is rebroadcast.

Location-based flooding uses a RAD timer and a coverage threshold that indicates the minimal additional coverage area that a rebroadcast of a packet has to cover. The additional coverage area is the area not covered by nodes that recently transmitted to the node. When a node receives a previously unseen packet it caches the packet and the location of the sender, and starts a RAD timer. Every time the node receives a redundant broadcast of the packet, the location of the sender is cached. When the RAD timer expires the additional coverage by a rebroadcast of the packet by the node is calculated. If the additional coverage is below the threshold, the packet is discarded. We measure additional coverage in percentage of the coverage area of the node.

The five flooding protocols presented above have been implemented in our NS-2 simulation model and table 2 summarises their parameters. The maximum value of the RAD timer has been set to 0.01 seconds [14]. We have not implemented flooding protocols that directly uses neighbour knowledge such as self-pruning [9] and multipoint relaying [13]. The reason is that the topology of vehicles travelling on a road is expected to frequently change, in particular on roads with opposing and multiple 
lanes, but also when passing intersections and access roads. This suggests that such methods might be counter-productive in terms of overhead. We have therefore left it as future work to evaluate neighbour knowledge methods in the context of the LIWAS system.

\begin{tabular}{lll}
\hline Flooding protocol & Parameter & Range \\
\hline \hline Probabilistic & Forwarding probability & {$[0 . .100] \%$} \\
Counter-based & Counter threshold & {$[2 . .9]$} \\
Distance-based & Distance threshold & {$[0 . .45] \mathrm{m}$} \\
Location-based & Coverage threshold & {$[0 . .55] \%$} \\
\hline \hline
\end{tabular}

Table 2: Flooding protocol parameters.

\section{Evaluation of Zone Flooding}

This section presents the main results obtained by simulations of the NS-2 model. Section 4.1 presents the initial simulation results investigating each zone flooding protocol in terms of transmissions and warned nodes. Section 4.2 investigates the impact of node speed and density on the performance of the protocols, and section 4.3 considers the size of the flooding zone. Section 4.4 investigates the protocols when node speed and interval are randomised.

\subsection{Basic Protocol Profiles}

The profile of each zone flooding protocol was investigated with the vehicle speed set to $30 \mathrm{~m} / \mathrm{s}$, the node interval to 20 meters, and the flooding zone to 1000 meters in each direction. The number of nodes was 200. Ten simulations were conducted for each configuration considered and we present the average result obtained. The standard deviation was insignificant in all cases.

For the above parameters the percentage of warned nodes was $100 \%$ for simple flooding using an average of 8532 transmissions. Figure 3 and Figure 4 show the corresponding numbers for probabilistic flooding as a function of the forwarding probability. Figure 4 shows that the percentage of warned nodes as expectedly increases when the forwarding probability is increased. A forwarding probability of $40 \%$ seems to be a good value for the forwarding probability as it yields close to 100 $\%$ of warned nodes while giving a significant reduction in the number of transmissions compared to simple flooding.

Figures 5-7 show the number of transmissions for counter-based, distance-based, and locationbased flooding as a function of the counter, distance, and coverage threshold, respectively. Figure 5 shows that the number of transmissions reaches its maximum when the counter threshold is 7 . This means that when the counter threshold is bigger than 6 , a node receives an average of 7 copies before the RAD timer expires. The percentage of warned nodes is $100 \%$ when the counter threshold is greater than or equal to 2 . When the counter threshold is 1 the number of warned nodes in $0 \%$ since a threshold of 1 implies that packets are always discarded. A counter threshold of 2 is optimal in this case.

The transmissions decreases smoothly for distance-based flooding except for the transitions from a threshold of 19 to 20 meters, and 39 to 40 meters where the transmissions decreases faster. The big difference between 19 to 20 is attributed to the node interval. With the parameters used, a node has always a neighbour at a distance of 20 meters. If the distance threshold is 19 meters that neighbour will always be further away than the distance threshold. Packets received from such a neighbour are 


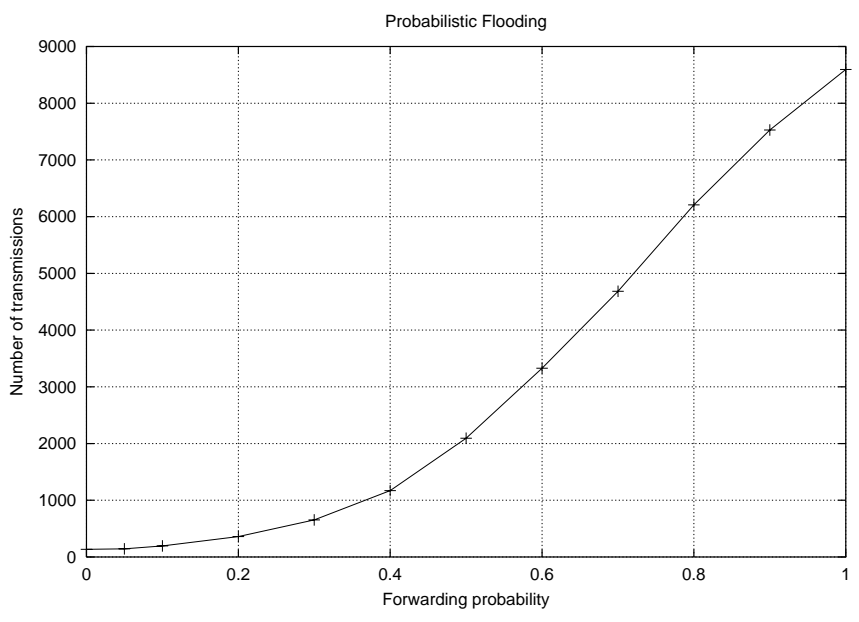

Figure 3: Performance of probabilistic flooding - transmissions.

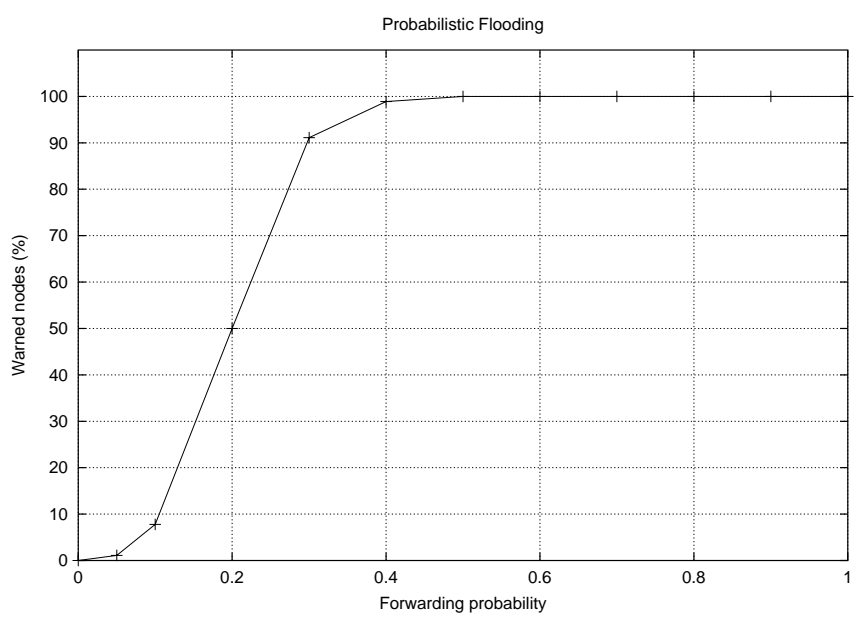

Figure 4: Performance of probabilistic flooding - aware nodes.

not discarded. If the distance threshold is 20 , then such packets will be discarded. The same happens at the transition from 39 to 40 . For the later comparison of the flooding protocols we will use a distance threshold of 35 meters. A threshold of 40 meters performs best because the positioning and movement of the nodes is symmetric. We have however selected a threshold of 35 meters since we will later randomise the node speed and interval. We do not show a graph with warned nodes, as the percentage of warned nodes is $100 \%$ when the distance threshold is less than or equal to 40 meters. If the threshold is bigger the percentage drops down to $0 \%$. This is expected since with a radio range of 40 meters, packets will always come from nodes located closer than the threshold in that case.

Figure 7 shows the number of transmissions for location-based flooding. The percentage of warned nodes is $100 \%$ when the threshold is less than or equal to $50 \%$. If the threshold is higher than $50 \%$, all packets received will be discarded, and packets will never be forwarded. For the later comparisons we will use 35\% as threshold for location-based flooding. Our results show there are thresholds that perform better, e.g., $45 \%$. We choose $35 \%$ because our later evaluations will also be 


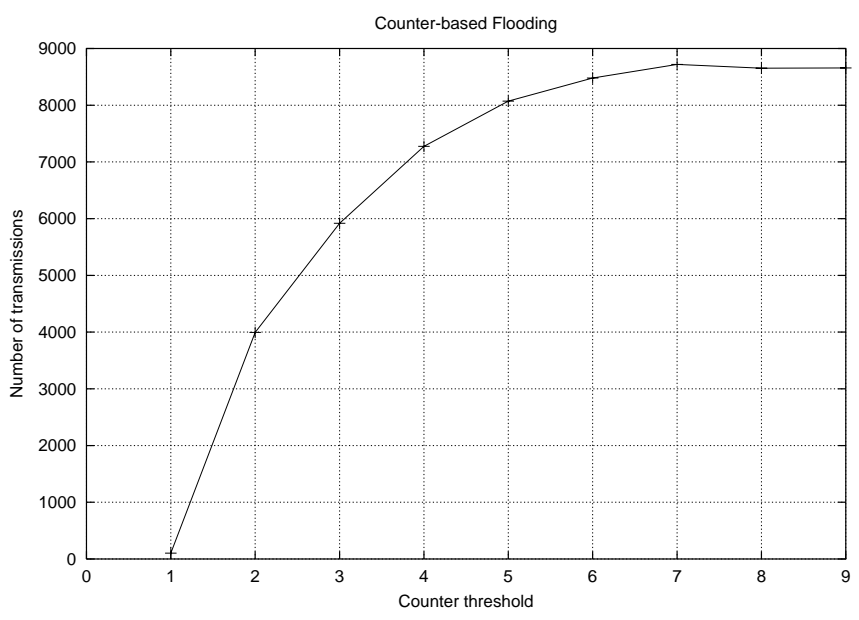

Figure 5: Transmissions in counter-based.

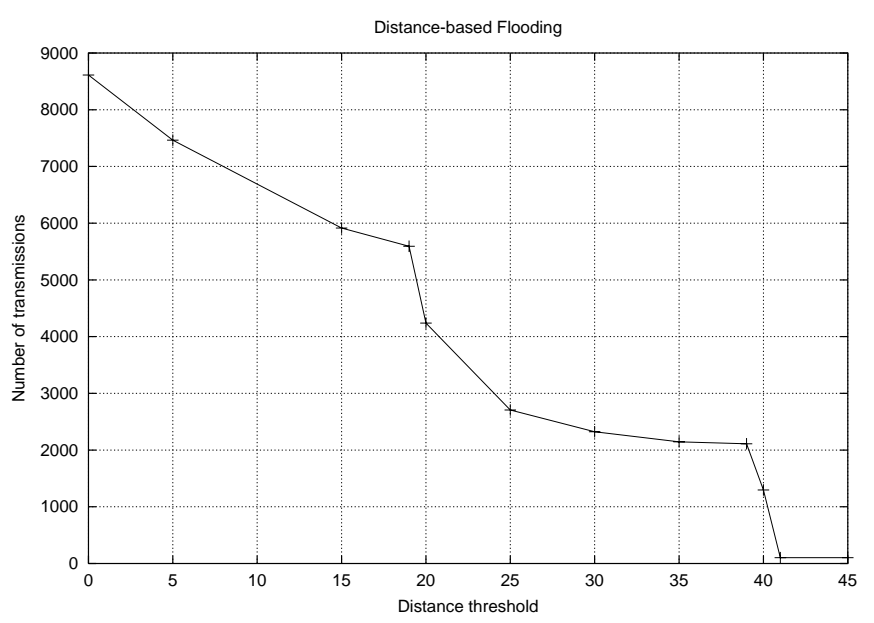

Figure 6: Transmissions in distance-based.

based on random node interval and speed. Since this creates node clustering, we believe that higher thresholds will be less effective. Location-based flooding has similar points as distance-based flooding where the number of transmissions decreases rapidly. This is caused by similar reasons as for distance-based flooding.

\subsection{Node Speed and Density}

The node speed had little impact on the performance of the zone flooding protocols. Node speeds in the interval from $10 \mathrm{~m} / \mathrm{s}$ to $30 \mathrm{~m} / \mathrm{s}$ were investigated, and the percentage of warned nodes did not change compared to the results presented in section 4.1. The variation in the number of transmissions was also insignificant. A main reason for this is that the transmission and propagation speed of packets are much faster compared to the speed of the nodes. For an example, in simple flooding it takes 0.13 seconds from the time a node registers ice on the road to the time when all nodes in the flooding zone 


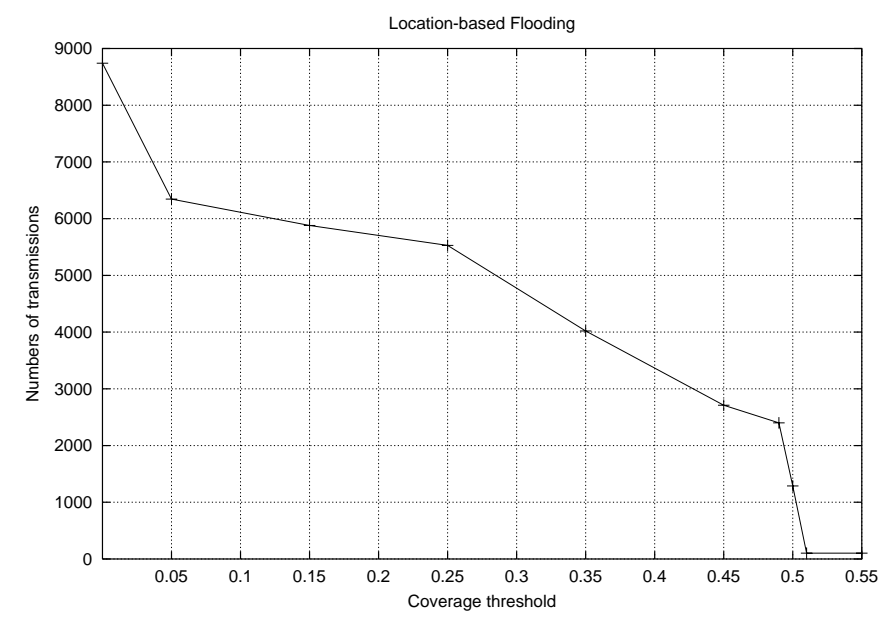

Figure 7: Transmissions in location-based.

have received the observation. In 0.13 seconds a node moving at $30 \mathrm{~m} / \mathrm{s}$ has only moved 4 meters. Taking into account that the radio range of a node is 40 meters, these 4 meters are insignificant. Furthermore, in the scenarios with a node interval of 20 meters there are no partitioning in the network. This shows that in the considered mobility scenario, the protocols are insensitive to node speed.

The node density affects the performance of the zone flooding protocols. Figures 8 and 9 show the impact of node interval for simple flooding (SF), probabilistic flooding (PF), and counter-based flooding (CF). The number of transmissions in simple flooding is 32200 when the node interval is 10 meters. This is very high in comparison with the 12292 in counter-based and the 8506 in probabilistic flooding. The number of transmissions decreases rapidly when the node interval increases. The decrease in transmissions is expected since when the node interval get larger, the number of nodes decreases. At a node interval of 25 meters the number of transmissions in probabilistic and counterbased flooding settles, while simple flooding still decreases. Counter-based flooding has a small increase when the node interval is 20 meters. This effect may have been caused by the symmetric location and movement of nodes. Probabilistic flooding and counter-based flooding perform similarly, with probabilistic flooding being slightly better in transmissions, but not as good in warned nodes.

Figure 9 shows that the percentage of warned nodes in simple and counter-based flooding is $100 \%$ for all node intervals. Probabilistic flooding decreases to $67 \%$ warned nodes when the node interval is 25 meters, and further down to $2 \%$ when the node interval is over 40 meters in which case packets are only forwarded by nodes in the opposing lanes. This shows that probabilistic flooding is rather sensitive to the node density.

Figures 10 and 11 show the impact of node interval on distance-based flooding (DF), where we have re-plotted the figures for simple flooding (SF) and counter-based flooding (CF) for comparison. The close relationship between the number of transmissions and the percentage of warned nodes is noticeable for distance-based flooding. The percentage of warned nodes is very poor when the number of transmissions is low. In distance-based flooding the number of transmissions does not decrease systematically as for the other protocols. Instead it behaves irregularly by being high for some node densities and low for others. For example, if the node interval is between 14 and 17 meters the number of transmissions is averaged to 180 , but when the interval is 18 meters the number of transmissions increases up to 2860. The reason for this behaviour is that for certain combinations of 


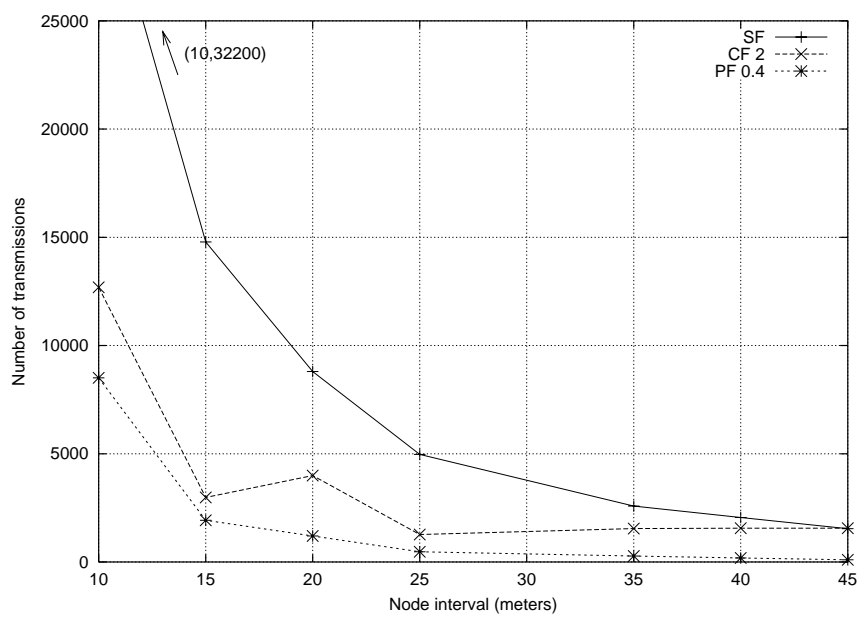

Figure 8: Impact of node density on transmissions in simple, probablistic, and counter-based flooding.

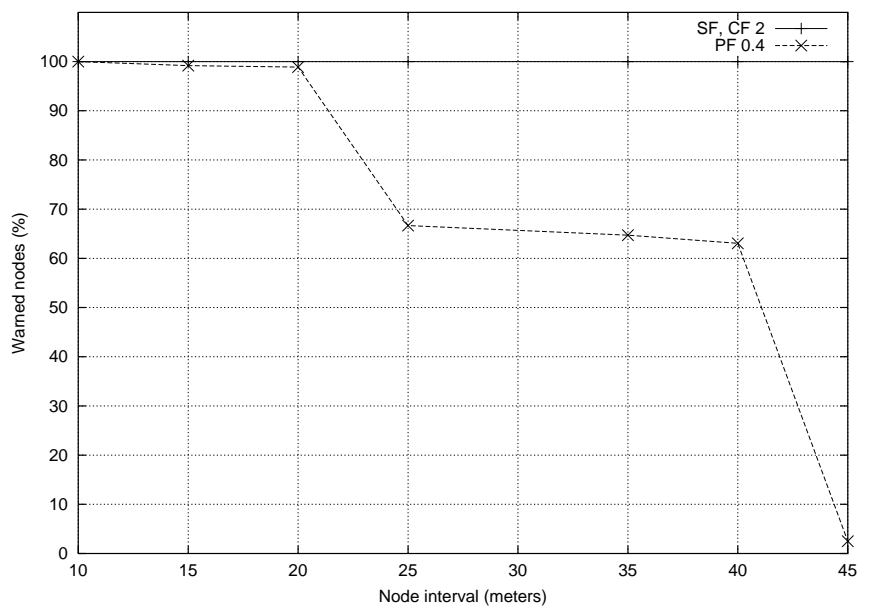

Figure 9: Impact of node density on aware nodes in simple, probablistic, and counter-based flooding.

node intervals and thresholds, all nodes within transmission range of a node are also within distance threshold. Hence, nodes that receive a packet from such a node are not able to forward it, causing the number of transmissions to drop. This is an unfortunate property of distance-based flooding.

The results for location-based flooding have been omitted as they are very similar to those obtained for distance-based flooding. In particular, they show a similar pattern of irregularities as distancebased flooding. The reasons are similar to those for distance-based flooding.

\subsection{Flooding Zone}

Figures 12 and 13 show the number of transmissions and the percentage of warned nodes for different sizes of the flooding zone. Except for probabilistic flooding (PS), the number of transmissions decreases in all cases with the size of the flooding zone. This is expected since the number of forwarding nodes decreases with the size of the flooding zone. Simple flooding still transmits the most 


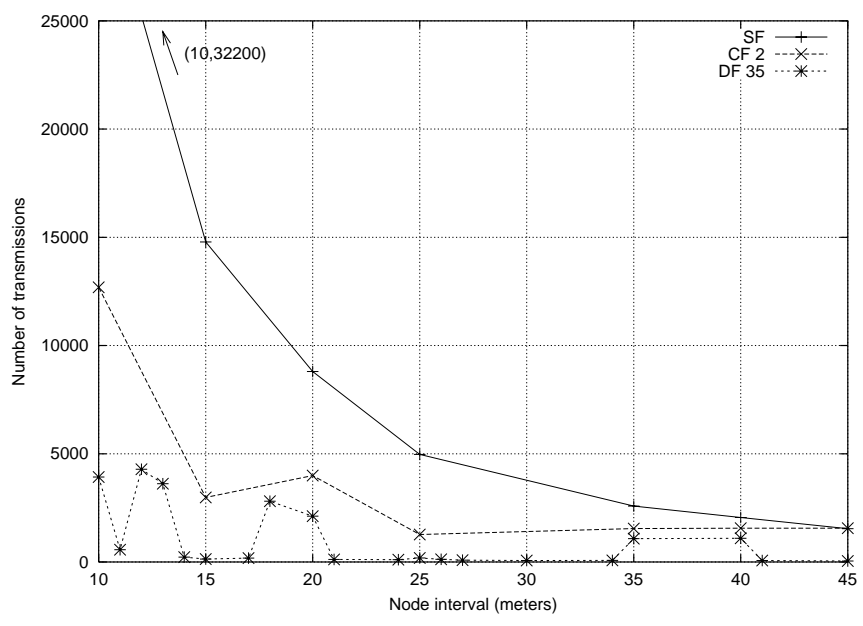

Figure 10: Impact of node density on transmissions in distance-based flooding.

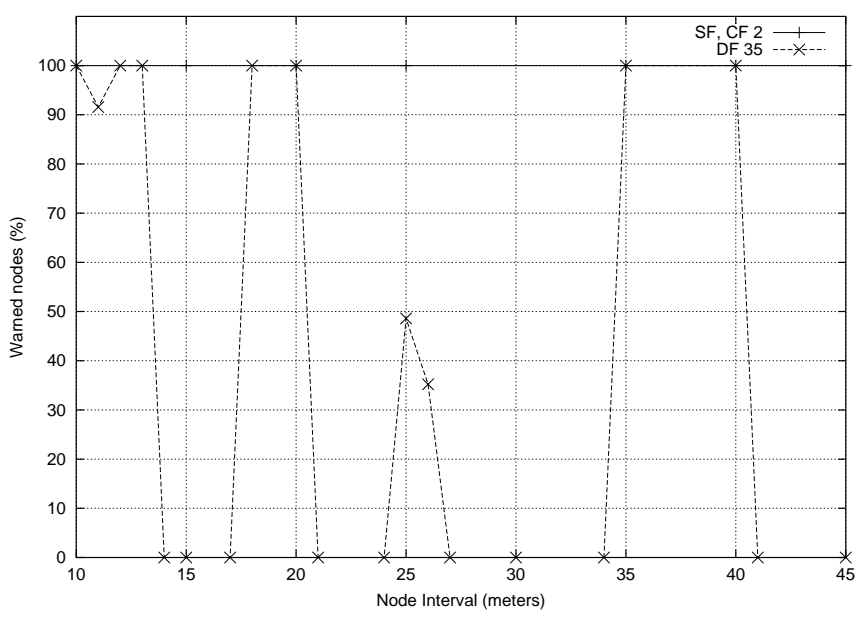

Figure 11: Impact of node density on aware nodes in distance-based flooding.

packets. Counter-based and location-based flooding follow close to each other. Distance-based and probabilistic flooding are those that perform best. The number of transmissions in probabilistic flooding are essentially constant. This is attributed to the fact that even when the flooding zone is large, packets do not travel many hops in probabilistic flooding, but enough hops to warn the vehicles in time. Considering the percentage of warned nodes, all protocols, except for probabilistic flooding, perform optimally until reaching a flooding zone size of 300 meters. When the flooding zone is 200 meters, the percentage decreases to $0 \%$ since it is then impossible to send packets to nodes outside the warning zone.

\subsection{Random Node Speed and Interval}

The results presented above were based on mobility scenarios with identical distance between nodes travelling at the same speed. To break this symmetry, we considered a mobility scenario where each 


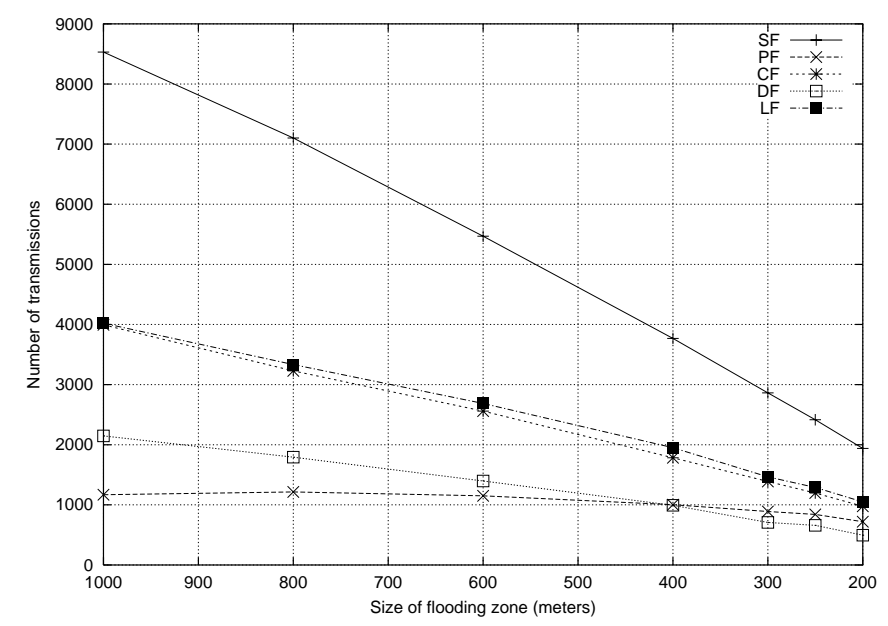

Figure 12: Impact of flooding zone size on transmissions.

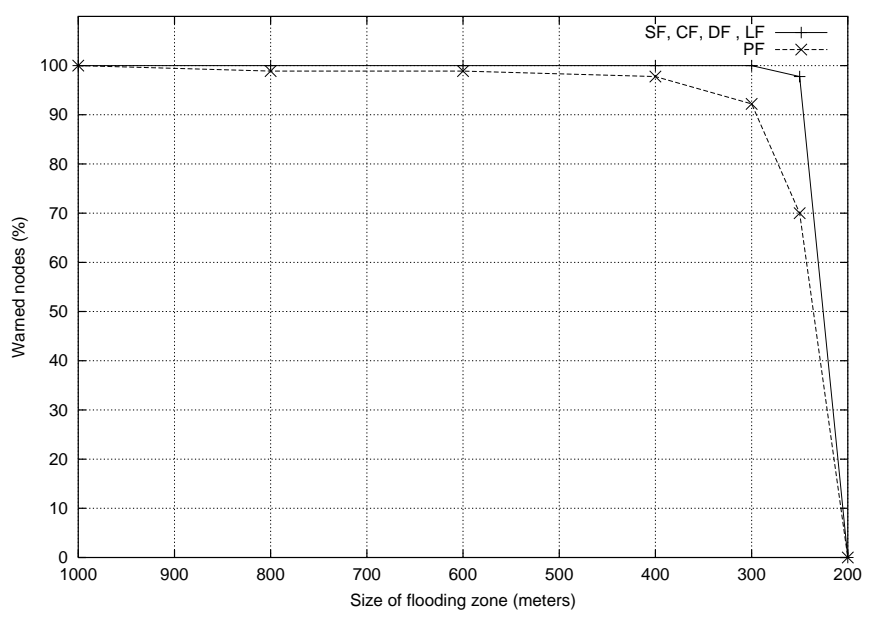

Figure 13: Impact of flooding zone size on aware nodes.

node is assigned a random speed, and nodes have random distances to each other in the range between 0 and 50 meters. Table 3 (left) shows the impact on the number of transmissions for the five protocols when evaluated in this randomised scenario. The table shows the number of transmissions and the reduction in percentage compared to using the identical node interval (20 meters) and speed (30 $\mathrm{m} / \mathrm{s}$ ) for all nodes. When using random interval and speed, the number of transmissions is reduced for all protocols. This is attributed to network partitioning which was not possible in the previous simulations. Table 3 (right) shows the percentage of warned nodes and the reduction in warned nodes for each protocol when using random node interval and speed. The most affected protocols are distance-based and location-based flooding. Both are sensitive to clustering because their threshold is based on distance. If the clustered nodes are too close to each other, packets will not be forwarded. The percentage of warned nodes in distance-based is very poor, $20 \%$, while location-based performs better, $82 \%$. In counter-based, the number of transmissions are reduced by $70 \%$ while still giving a high percentage of warned nodes, $96 \%$. Simple flooding shows a small reduction in transmissions, 


\begin{tabular}{l|rr||rr}
\hline Protocol & Transmissions & Reduction & Warned nodes & Reduction \\
\hline \hline Simple Flooding & 5013 & $41 \%$ & $98 \%$ & $1 \%$ \\
Probabilistic & 695 & $41 \%$ & $66 \%$ & $34 \%$ \\
Counter-based & 1202 & $70 \%$ & $96 \%$ & $4 \%$ \\
Distance-based & 210 & $91 \%$ & $20 \%$ & $80 \%$ \\
Location-based & 886 & $88 \%$ & $82 \%$ & $18 \%$ \\
\hline \hline
\end{tabular}

Table 3: Performance in scenario with randomised node speed and interval.

$41 \%$, and still has an outstanding percentage of warned nodes, $98 \%$. Probabilistic flooding has the smallest number of transmissions and a moderate $66 \%$ of warned nodes.

\section{Conclusions and Future Work}

We have presented the concept of zone flooding and performed an initial evaluation in the context of the LIWAS traffic warning system, comparing five flooding protocols for implementation of zone flooding.

The work on evaluation of the zone flooding protocols have provided valuable insight into the operation of the protocols, in particular their robustness to change in various parameters. The act of constructing the simulation model and conducting the performance evaluation has also given ideas and direction for future work on the LIWAS communication infrastructure. In addition to the constructed simulation model, we are currently implementing some first small-scale prototypes of the LIWAS system based on laptops and 802.11 radios.

The results presented in this paper have clearly highlighted the trade-off between transmissions and percentage of warned nodes. The investigations showed that location-based and distance-based flooding are highly sensitive to clustering and node density, and that this may lead to unacceptable performance in terms of warned nodes. Probabilistic flooding showed a similar problem in randomised scenarios. Simple flooding puts a heavy load on the network, and based on our investigations, a counter-based protocol is currently the best candidate. The investigations in [14] concluded that location-based flooding performed better than counter-based flooding. As stated initially, we evaluate the protocols in mobility scenarios that are very different from [14]. Furthermore, we use warned nodes rather than delivery ratio as an evaluation criteria. A common problem for all the advanced flooding protocols is that their performance heavily depends on the choice of parameters for a given mobility pattern. Since vehicles equipped with the LIWAS system will be exposed to different mobility patterns, it seems that adaptive versions of the advanced flooding protocols are required to achieve satisfactory performance in all cases. The design of adaptive flooding protocols is future work.

The presented protocols assumes a GPS device to implement the flooding zone. It would be interesting to investigate whether this assumption can be removed using alternatives such as hopcount or time-to-live to limit the flooding. The presented protocols do not all achieve a percentage of $100 \%$ in warned nodes while having relatively few transmissions. Investigating modifications to further improve the percentage of warned nodes is therefore of interest. Finally, our application of zone flooding assumes that vehicles are repeatedly passing the icy section to initiate new flooding and warn approaching vehicles. Lifting this assumption is future work.

The presented NS-2 simulation model constitute the first step in an evaluation of the communication infrastructure in the LIWAS system. The LIWAS system will eventually include a combination of 
vehicles with sensors, road-signs, and stationary sensors. The plan is to continue the development of the NS-2 model along-side the development, implementation, and deployment of the LIWAS system.

Acknowledgements. The work described in this paper has been supported by ISIS Katrinebjerg Software and the Danish Natural Science Research Council.

\section{References}

[1] Bogenberger et. al. Virtual City Portal - A Multi-Network Personal Information System for Automobile Users. In Proc. of Workshop on Multiradio Multimedia Communications, Communication Technology for Vehicles, 2004.

[2] R. Morris et. al. CarNet: A Scalable Ad Hoc Wireless Network System. In Proc. of the 9th ACM SIGOPS European workshop, 2000.

[3] S. Ni et. al. The Broadcast Storm Problem in a Mobile Ad Hoc Network. In Proc. of ACM/IEEE MobiCom'99, pages 151-162, 1999.

[4] T. Nadeem et. al. TrafficView: A Scalable Traffic Monitoring System. In Proc. of IEEE International Conference on Mobile Data Management, pages 13-26, 2004.

[5] W. Franz et. al. Internet on the Road via Inter-Vehicle Communications. In Proc. of Workshop der Informatik 2001:Mobile Communications over Wireless LAN, 2001.

[6] T. Imielinski and J.C. Navas. GPS-based Geographic Addressing, routing, and Resource Discovery. Communications of the ACM, 42(4):86-92, 1999.

[7] Invent. www.invent-online.de.

[8] Young-Bae Ko and Nitin H. Vaidya. Flooding-based Geocasting Protocols for Mobile Ad Hoc Networks. Mobile Networks and Applications, 7:471-480, 2002.

[9] H. Lim and C. Kim. Multicast Tree Construction and Flooding in Wireless Ad Hoc Networks . In Proc. of ACM Int. Workshop on Modelling, Analysis, and Simulation of Wireless and Mobile Systems, 2000.

[10] LIWAS: Life Warning Systems. www.liwas.dk. (in danish).

[11] The network simulator - ns-2. www.isi.edu/nsnam/ns/.

[12] C.E. Perkins. Ad Hoc Networking. Addison-Wesley, 2001.

[13] Amir Qayyum, Laurent Viennot, and Anis Laouiti. Multipoint Relaying: An Efficient Technique for flooding in Mobile Wireless Networks. Technical Report Research Report RR-3898, INRIA, February 2000.

[14] B. Williams and T. Camp. Comparison of Broadcasting Techniques for Mobile Ad Hoc Networks. In Proceedings of the ACM International Symposium on Mobile Ad Hoc Networking and Computing, pages 194-205, 2002. 